\title{
Special issue on information visualisation
}

\author{
Rita Francese ${ }^{1} \cdot$ Ebad Banissi $^{2} \cdot$ Michele Risi $^{1}$ \\ Published online: 29 October 2019 \\ (C) Springer Science+Business Media, LLC, part of Springer Nature 2019
}

We are in the Big Data era, characterized by an increasing amount of information generated everyday by all the phenomena concerning our life. These data are collected by many information sources and systems ranging from IoT sensors, large databases, social networks and interactive platforms, as well as generic media like images, videos and audios. Thus, to be able to comprehend the phenomena underlying this data there is the need of designing effective visualization tools.

The papers selected for this special issue outline and evaluate some recent works on multimedia tools for information visualization and interaction with various types of data, including textual documents, graphs, spatio-temporal data, multimedia contents, and musical data.

The international conference on information Visualisation (iV), one of the longest running on the subject has open forum philosophy of interdisciplinary drive to define its subject theme and discussion and focus group. This special issue of Multimedia Tools and Applications for Information Visualisation is based on the handful of submissions to the $22^{\text {nd }}$ International Conference on Information Visualisation (iV18), which was held in Italy in July 2018. The selected papers give examples of techniques, domains and number of case studies to address some issues.

The papers presented in this Special Issue investigate different aspects of the theme highlighted above.

The first set of papers $[1,2,12,16]$ investigate and evaluate the visualization of spatial, temporal and spatio-temporal data. Almeida et al. [2] consider how and why consideration of Level of Detail (LoD) plays a crucial role in analytics. They propose a Visual Analytics approach - VAST, allows users to simultaneous inspection of phenomenon using different LoDs. VAST demonstrates using LoDs will reveal different prospective and hence portraying different perception of the phenomenon.

Rita Francese

francese@unisa.it

Ebad Banissi

banisse@1sbu.ac.uk

Michele Risi

mrisi@unisa.it

1 University of Selarno, Salerno, Italy

2 London South Bank University, London, UK 
Virtual Reality (VR) technologies are adopted by Okada et al. [16] to perform a spatio-temporal social media data exploration by three-dimensional temporal visualization of tweets of microblogs with location information. They further highlight how VR can used to explore and extract further details tweets of microblogs contents as well as t-temporal attributes of the events.

Afonso and et.al. [1] explore analytics in video games by using visualizations techniques, in particular animated maps, for control and analysis of spatio-temporal information data associated to player performance within game environment.

Garcia et Al. [12] present a collaborative virtual reality environment in which distributed multidisciplinary teams involved in space exploration activities come together and explore areas of scientific interest of a planet for future missions.

The evolution from low to high dimension data requires new approaches for detecting functional dependencies between different dimension. Caruccio et al. [7] propose a visual metaphoric approach for visualizing approximate functional dependencies for big- and multimedia- data.

Malandrino et Al. [15] and Coletta and et al. [9] focu on the limitations of modern music composition tools. Malandrino et Al. [15] highlight the problem of learning and compose a specific type of classical music: music in chorale style. They further demonstrates how additional (visual) information, provided through a specific software tool, could be useful to improve learning and composition of this kind of music.

Coletta and et.al. [9] demonstrate how the natural channels of communication as used by an orchestra conductor can be mapped as gesture- and voice-based multimodal wrapper for music editor applications. The wrapper translates user gestures and music jargon keywords into mouse clicks or keyboard touch-actions.

The next two papers $[3,14]$ propose new techniques and approaches to improve the visualization of multidimensional graphs. Bruder et al. [3] examine the visualization and interactive analysis of dynamic graphs that contain a large number of time steps of time varying data. Dynamic graph are represented by space-time cubes by stacking the adjacency matrices.

The visualization of undirected graphs and symmetric square matrices are complex, especially for high dimensional graph. A method for visualizing undirected graphs and symmetric square matrices is proposed in [14]. Graphs are transformed into overlapping sets, which in turn visualized by exploiting a set visualization techniques such as Euler diagrams or rainbow boxes.

Visualization techniques are often adopted in Software Engineering for different purposes. In this special issues, two papers are related to software visualization and multimedia control. Software visualization is to aid comprehension technique adopted in the context of software maintenance, reverse engineering, and software evolution analysis. Romano et al. [18] adopts city metaphor, where a program is represented by a city and evaluate its implementation in both a VR-based tool and a 3D-based tool with respect to users' feelings, emotions, and thinking.

Revision control has been widely deployed for text files, but can be inconvenient when applied for multimedia applications because of significant amount of binary data, such as images, videos, meshes, and animations. Castellano et al. [5] propose RECODE, a revision control system for digital images which stores revisions in the form of a directed acyclic graph, where nodes represent editing operations, and edges describe the spatial and temporal relationships between operations.

The rapid growth of multimedia application needs the development of new visualization techniques for analyzing them from different perspectives. Del Fatto et al. [11] present a 
multimedia analytics application for summarizing and analyzing temporal data captured by eye-tracking experiments, while Koivunen-Niemi [13] proposes the use of temporal visualizations for examining differences in media narrative patterns over time and across publications. In particular, they compare co-occurrences of variables in multimedia content chronologically, which can be more useful in identifying patterns and possible biases in media coverage than simply counting the individual occurrences of those variables independently. The paper proposes a visualization approach, called time-sets, aiming at support the temporal comparisons of such co-occurrences.

The remaining papers in this special issue stand in between theoretical and applicative approaches, by exploring novel applications of visualization techniques.

The scope of the paper proposed by Carrion et al. [6] concerns Taxonomies, semantic resources that help to categorize and add meaning to data by extracting knowledge from large and unstructured data sets. The creation of Taxonomies is very onerous and requires the intervention of domain experts. The paper proposes a semi-automatic taxonomy generation tool for supporting domain experts in building taxonomies that used to automatically create semantic visualizations of data.

Corbatto \& Dattolo [10] examine the problem of providing support to teachers which have to conduct their lessons having not only the availability of a huge amount of contents and learning objects on the Web.

Cattaneo et al. [8] investigate the Source Camera Identification problem (i.e., determining the particular digital camera used for taking a given image) on Hadoop, a software framework that provide coding of distributed applications starting from a MapReduce algorithm with little programming efforts.

When users are immersed in a Virtual Reality environment their perception is affected by the environment stimuli. Perales et al. [17] create an interactive VR system which combines the power of VR with other multimedia technologies such as sounds (binaural), music or colors, to study how they affect moods and also to the brainwaves and how they are sensed by the users. To evaluate the system Electro-Dermal Activity is adopted to compare subjective user perceptions against physical parameters variations.

Buono and et al. [4] propose an analysis of air quality data, which may reveal the quality of life and can prevent dangers for the citizen health. This work presents a visual analytics approach to support air quality experts in the analysis of data produced by electronic nose devices. Clustering and visualization techniques are adopted to analyze such data with the support of calendar, map and line graph visualization.

We conclude this editorial with special thanks to both the authors, who decided to share their experience and research, and to the reviewers, who provided very helpful suggestions to clarify and improve the papers. We hope the readers will enjoy the papers collected in this special issue and get inspiration for new ideas and research directions.

The Guest Editors

\section{References}

1. Afonso AP, Carmo MB, Goncalves T, Vieira P (2019) VisuaLeague: Player Performance Analysis using Spatial-temporal Data

2. Almeida Silva R, Moura Pires J, Datia N, Santos MY, Martins B, Birra F. (2019) Visual Analytics for SpatioTemporal Events 
3. Bruder V, Lahmar HB, Hlawatsch M, Frey S, Burch M, Weiskopf D, Herschel M, Ertl T (2019) VolumeBased Large Dynamic Graph Analysis Supported by Evolution Provenance

4. Buono P, Balducci F (2019) MonitorApp: a Web Tool to Analyze and Visualize Pollution Data Detected by an Electronic Nose

5. Calefato F, Castellano G, Rossano V (2019) RECODE: Revision Control for Digital Images

6. Carrion B, Onorati T, Díaz P, Triga V (2019) A Taxonomy Generation Tool for Semantic Visual Analysis of Large Corpus of Documents. Onorati

7. Caruccio L, Deufemia V, Polese G (2019) Visualization of (Multimedia) Dependencies from Big Data

8. Cattaneo G, Ferraro Petrillo U, Abate A F, Narducci F, Barra S (2019) Achieving Efficient Source Camera Identification on Hadoop

9. Coletta A, De Marsico M, Panizzi E, Prenkaj B, Silvestri D (2019)MIMOSE: Multimodal Interaction for Music Orchestration Sheet Editors

10. Corbatto M, Dattolo A (2019) Exploring AppInventory, a visual catalog of applications for assisting teachers and students

11. Del Fatto V, Dignos A, Raimato G, Maccioni L, Borgianni Y, Gamper J (2019) Visual Time Period Analysis: a Multimedia Analytics Application for Summarizing and Analysing Eye-tracking ExperimentsVincenzo Del Fatto

12. Garcia AS, Fernando T, Roberts DJ, Bar C, Cencetti M, Engelke W, Gerndt A (2019) Collaborative Virtual Reality Platform for Visualizing Space Data and Mission Planning

13. Koivunen-Niemi L, Masoodian M (2019) Visualizing narrative patterns in online news media

14. Lamy JB (2019) Visualizing undirected graphs and symmetric square matrices as overlapping sets: Methods and application to character co-occurrences graphs and matrices in novels and DBpedia

15. Malandrino D, Pirozzi D, Zaccagnino R (2019) Learning the Harmonic Analysis: Is visualization an effective approach?

16. Okada K, Yoshida M, Itoh T, Czauderna T, Stephens K (2019) VR System for Spatio-Temporal Visualization of Tweet Data and Support of Map Exploration

17. Perales F J, Riera L, Ramis S, Guerrero A (2019) Evaluation of a VR system for Pain Management using binaural acoustic stimulation

18. Romano S, Capece N, Erra U, Scanniello G, Lanza M (2019) The City Metaphor in Software Visualization: Feelings, Emotions, and Thinking

Publisher's note Springer Nature remains neutral with regard to jurisdictional claims in published maps and institutional affiliations. 